\title{
A Case of Cyclopentolate Induced Psychosis
}

\author{
${ }^{1}$ Vishnu Vardhan, ${ }^{2}$ Sharath Vishwaraj
}

\begin{abstract}
Cyclopentolate is the cycloplegic drug of choice in children older than 1 year. Though rare, if absorbed systematically, it is known to cause Central Anticholinergic Syndrome. Children are especially prone due to their lower body weight. Symptoms and signs in children include flushing, tachycardia, feeding intolerance, seizures, and drowsiness. We may also see behavioral changes and transient psychotic reactions. We describe a case in which the patient had acute behavioral and psychotic manifestation following instillation of cyclopentolate.
\end{abstract}

Keywords: Central anticholinergic syndrome, Cyclopentolate, Psychosis.

Key message: Central Anticholinergic Syndrome, though rare, can occur following topical instillation of cyclopentolate and other mydriatic agent. Clinicians using cyclopentolate must be aware of it.

How to cite this article: Vardhan V, Vishwaraj S. A Case of Cyclopentolate Induced Psychosis. J Med Sci 2016;2(2):36-37.

Source of support: Nil

\section{Conflict of interest: None}

\section{INTRODUCTION}

Central anticholinergic syndrome (CAS), produced by over dosage or abnormal reaction to clinical dosage of anticholinergic drugs, was first described by Longo in 1966. Systemic absorption of the drug, following eye drop instillation, can occur transconjunctivally or via nasolacrimal duct through the highly vascular nasal mucosa. ${ }^{1}$ Complications are seen in up to $10 \%$ of cases. These include tachycardia and central nervous system (CNS) effects like restlessness, hallucination, psychosis, hyperactivity, seizures, incoherent speech, and ataxia., ${ }^{2,3}$ We describe a case in which the patient had acute behavioral and psychotic manifestation following instillation of cyclopentolate.

\section{CASE REPORT}

We report a case of a 10-year-old school-going girl, who presented with diminution of vision in both the eyes, not

\footnotetext{
${ }^{1}$ Associate Professor, ${ }^{2}$ Junior Resident

1,2Department of Psychiatry, RajaRajeswari Medical College and Hospital, Bengaluru, Karnataka, India
}

Corresponding Author: Vishnu Vardhan, Associate Professor Department of Psychiatry, RajaRajeswari Medical College and Hospital, Bengaluru, Karnataka, India, e-mail: myvish9@gmail. com, sharathvishwaraj@gmail.com able to clearly see the board. She had no history of allergy, systemic illness, or drug intake in the past. She was being investigated for refractive error, and cyclopentolate eye drops $1 \%$ was used for dilatation of pupil. Patient's father was advised to instill 2 drops in each eyes, two times at 15 minutes interval. Inadvertently, the father instilled it five to six times within a period of 2 hours. After 1 hour of the last dose, she was referred to Psychiatry Outpatient Department with altered behavior in the form of inappropriate laughing and disinhibited behavior, fearfulness, irritability, and visual hallucination. On examination, her pupils were widely dilated and fixed. She displayed emotional lability and irrelevant speech with increase in psychomortar activity. Mental status examination revealed visual hallucinations of seeing plate, noodles, and chips packet. Other examination was unremarkable. Based on these findings and their temporal relation to overdose of cyclopentolate eye drops, a diagnosis of cyclopentolate toxicity was made. She was treated symptomatically and her symptoms gradually disappeared over the next 8 to 10 hours. She was discharged after 24 hours of observation.

\section{DISCUSSION}

Brain has wide distribution of acetylcholine with its receptors and is important in regulating many functions, including the sleep-wake cycle, memory, alertness, orientation, and analgesia. An absolute or relative reduction in cholinergic activity in the CNS can result in CAS with various signs and symptoms. ${ }^{4}$ They include CNS excitation or depression, which manifest as excitation, convulsions, hallucinations, disorientation, hyperpyrexia, hyperalgesia, ataxia, and mental impairment. This may end in sedation, and coma. ${ }^{5}$ The drug cyclopentolate has been widely used as a cycloplegic and mydriatic agent for number of years. It is a first choice for most children over the age of 1 year, and allows successful cycloplegic refractions with few complications. ${ }^{2,4,5}$ This drug is an anticholinergic and antimuscarinic with atropine-like actions. If it is instilled topically in the eye, it is well absorbed, locally and systemically. The route of systemic absorption is through the conjunctiva, the nasolacrimal duct, the oropharynx, the digestive system, and the skin. In our case, as seen in other pediatric cases, cyclopentolate eye drops could well have been absorbed by conjunctival capillary and reached the brain via angulus venosus of 
deep cerebral veins and cavernous sinuses. ${ }^{4,5}$ Reports of systemic toxicity following topical application of cyclopentolate eye drops are present in the English literature. Children are especially prone due to their lower body weight. In children it may present as flushing, tachycardia, feeding intolerance, seizures, and drowsiness. ${ }^{2,6,7}$ Behavioral changes and transient psychotic reactions may also occur. ${ }^{6,7}$ Commonly used drug is Physostigmine as antidote drug of choice. It readily crosses the bloodbrain barrier (BBB). Other anticholinesterases drugs like neostigmine, pyridostigmine, and edrophonium do not cross the BBB. Hence, they are not useful. ${ }^{5,8}$

\section{CONCLUSION}

The topical ophthalmic preparations are widely prescribed by ophthalmologists. We should prevent and reduce systemic absorption and toxicity. Our case report highlights the important side effects of a topically administered drug. Medical and paramedical staff should use the drug in the prescribed dose and strictly adhere to methods of administration, to minimize systemic absorption.

\section{REFERENCES}

1. Palmer EA. How safe are ocular drugs in pediatrics? Ophthalmology 1986 Aug;93(8):1038-1040.

2. Bhatia SS, Vidyashankar C, Sharma RK, Dubey AK. Systemic toxicity with cyclopentolate eye drops. Indian Pediatr 2000 Mar;37(3):329-331.

3. Mirshahi A, Kohnen T. Acute psychotic reaction caused by topical cyclopentolate use for cycloplegic refraction before refractive surgery: case report and review of the literature. J Cataract Refract Surg 2003 May;29(5):1026-1030.

4. Rajappa N, Patra S, Bhalsing S, Lune AA. A case of acute psychosis induced by topical cyclopentolate eye drops in an elderly patient. Med J DY Patil Univ 2014;7(1):68-69.

5. Pooniya V, Pandey N. Systemic toxicity of topical cyclopentolate eye drops in a child. Eye (Lond) 2012 Oct;26(10):1391-1392.

6. Lim DL, Batilando M, Rajadurai VS. Transient paralytic ileus following the use of cyclopentolate-phenylephrine eye drops during screening for retinopathy of prematurity. J Paediatr Child Health 2003 May-Jun;39(4):318-320.

7. Gray C. Systemic toxicity with topical ophthalmic medications in children. Paed Perinat Drug Ther 2006 May;7(1):23-29.

8. Pappano AJ, Katzung BG. Cholinoceptor-blocking drugs. In: Katzung B, editor. Basic and clinical pharmacology. 9th ed. New York (NY): McGraw-Hill; 2004. p. 109-121. 